\title{
Development of professional readiness of teachers to work with children exhibiting aggressive behavior
}

\author{
Olesya Istrofilova ${ }^{1, *}$ \\ ${ }^{1}$ Nizhnevartovsk State University, 630091, Nizhnevartovsk, Russia
}

\begin{abstract}
In the pedagogical theory and practice, there is a contradiction between the presence of a large number of aggressive school children and poor readiness of teachers to interact with aggressive teenagers. The paper presents the results of the experiment on the development of professional readiness of future teachers to work with children and adolescents exhibiting aggressive behavior.
\end{abstract}

\section{Introduction}

Aggression has become one of the sad characteristics of our century. Today, the statistical data on the growth of juvenile delinquency are not surprising. Adults sometimes do not know how to overcome growing teenage cruelty and hostility. There is no need to prove how important it is to find ways to cultivate peace in children. Many scientists and teachers are trying to solve this difficult task. One of such ways is training of teachers for interaction with children and adolescents who exhibit aggressive behavior.

The solution to many problems associated with providing assistance to children with aggressive behaviors, depends on the degree of real readiness of teachers to professional activities. The analysis of the problem of training specialists for educational work with adolescents exhibiting aggressive behavior allowed us to draw a number of conclusions:

- the theoretical search and practical implementation of the solution to the problem of "difficult" childhood developed by famous teachers of the 1920-1930s, are rethought in modern conditions;

- the difficult socio-economic situation contributes to the growth of various disharmonies of child's personality;

- researchers have developed methods for training students for interaction with adolescents with impaired behavior, but in most cases they relate to preschool and primary school children; less research is carried out on the behavior of adolescent school children;

- the problem of training future teachers for working with aggressive children and adolescents is important and requires special training;

- the condition for effective interaction of a teacher with adolescents with aggressive behavior is to equip them with knowledge and methods for organizing pedagogical diagnostics, prevention and correction.

The problem of professional readiness of future teachers to work with aggressive children and adolescents is part of the general problem of professional training of teachers. To date, a number of studies reveal theoretical foundations of the development of professional readiness of future teachers: I.A. Golubeva, K.M. Durai-Novakova [1], L.A. Ibragimova [2], R.S. Nagovitsyn [3], T.Ya. Yakovets et al. D.Zh. Azimova, L.A. Blokhina, V.A. Slastenin, L.I. Fedorova et al. deepened this topic and revealed theoretical foundations of training teachers for performing educational functions. Currently, a number of scientists have devoted research to the problem of training specialists for working with difficult children: V.N. Barsukov, S.A. Zavrazhin, V.N. Irkhin [4], G.M. Potanin, V.V. Trifonov [5], R.M. Khmurich et al. Theoretical and experimental works by A. Bandura [6], L. Berkovits, R. Baron, K. Butner, K. Lorenz, E. Erickson et al. are devoted to this issue. There are no studies on the system of training teachers for educational work with adolescents exhibiting aggressive behavior. However, it should be noted that a different approach to the development of professional readiness of teachers to work with these children is required.

\section{Main body}

Analysis of scientific views of K.I. Duray-Novakova, L.A. Ibragimova, T.Ya. Yakovets made it possible to conclude that quality of readiness for professional activity depends on methods of training. The purpose of training is to develop readiness for certain activities. Readiness for professional activities is considered as the goal of training and its result expressed in mastering knowledge, skills, gaining initial professional experience.

When developing a program of professional training of students for working with aggressive teenagers, we proceeded from the (personal) educational paradigm, ideas about the purpose, content and technologies of pedagogical activities, the different nature of the relationship between teachers and students.

The working with students within the author's program was aimed at creating theoretical, practical and

\footnotetext{
*Corresponding author: ol_istr@mail.ru
} 
psychological readiness for organizing work on the diagnosis, prevention and correction of aggressive behavior of adolescents as a required aspect of their professional and personal development. To achieve this goal, the following tasks were solved:

- formation of a positive motivational attitude towards readiness to work with aggressive teenagers as an urgent need;

- acquisition of the required and sufficient level of knowledge of medical, social, psychological, and pedagogical aspects of children who are prone to aggressive behavior;

- accumulation of experience of pedagogical interaction with adolescents with deviations in behavior; gradual formation of a system of skills aimed at organizing the communication of adolescents prone to aggressive behavior.

The hypothesis of the study is that the process of professional training of students to work with teenagers exhibiting aggressive behavior will be more effective and provide the required level of professional readiness to implement this activity, if they know psycho-pedagogical bases of aggressive behavior of teenagers and the following pedagogical conditions are met:

- the basis of the process of training future teachers for working with aggressive teenagers is a model for organizing work to correct aggressive behavior of adolescents;

- required knowledge for implementing the system of diagnosis, prevention and correction of aggressive behavior of adolescents;

- active forms and methods of teaching students how to interact with aggressive teenagers on the basis of knowledge of diagnostic, prognostic, socio-therapeutic tools, and correctional and pedagogical skills;

- diagnostic tools and pedagogical monitoring of the process of developing readiness of future teachers to work with aggressive children and adolescents [7].

When determining the list of psychological and pedagogical conditions, we adhered to the characteristics given by T.Ya. Yakovets. Pedagogical conditions are "a set of objective possibilities, forms, methods, pedagogical situations, circumstances and techniques aimed at solving assigned tasks" [8]. The implementation of pedagogical conditions contributes to the formation of all the components of readiness for this type of work and provides a sufficient level of its formation.

When developing a specialist training program, we proceeded from the accepted definition of readiness of a future teacher to work with aggressive teenagers. The willingness of students to work with aggressive adolescents is determined by the presence of relevant needs and personal qualities; knowledge of the theory and methods of pedagogical diagnostic and correctional work with such adolescents; skills to identify aggressiveness in adolescents and its elimination. This readiness includes the following structural components: motivationalpersonal, cognitive and practical, which are the criteria for professional readiness.

In accordance with the objectives of our study, based on the principles of the professiographic approach, we developed a program for training students for work with aggressive teenagers, including motivational-personal, cognitive and practical components. To characterize each component, we determined indicators and levels of formation of this readiness. The motivational-personal component is characterized by the need to master the method of interaction with adolescents exhibiting aggressive behavior, personal qualities of the teacher, the leading style of pedagogical communication, and ways of self-regulation. The cognitive component includes knowledge of the method of interaction with aggressive adolescents. The practical component assumes that students have organizational, diagnostic and correctional skills that can successfully eliminate aggressive manifestations in the behavior of adolescent children. The main components of the content of professional readiness to work with adolescents exhibiting aggressive behavior are interconnected and interdependent. Based on these criteria, the levels of formation of readiness were determined: high, medium, low.

\section{Results of experiments}

The experiment aims to develop professional readiness for working with aggressive teenagers as a certain system that cannot function in isolation from other, various aspects of the professional training.

The 2nd, 3rd and 4th year students of the Faculty of Pedagogy and Psychology of Nizhnevartovsk State University took part in the experiment (107 teachers, psychologists, deputy directors for educational work, social educators of secondary schools).

In accordance with the subject, the ascertaining stage of the experiment was aimed at solving the following tasks:

- analysis of professional readiness of teachers of educational institutions (Nizhnevartovsk) in order to establish the degree of formation of ideas about aggressive behavior and difficulties that arise when interacting with aggressive children and adolescents; - analysis of the theoretical training of university students in order to determine the level of knowledge and pedagogical skills required to establish effective interaction with children and adolescents exhibiting aggressive behavior.

The results of surveys of school teachers and university students suggest that there are general trends in identifying typical difficulties in interacting with adolescents exhibiting aggressive behavior - difficulties of understanding the essence of deviant behavior, the aggressive state of a person and forms of its manifestation, organization of diagnostic and preventive work with adolescents. The survey results showed that students do not have a clear understanding of aggression as a specific form of behavior or a response to adverse factors. Students have lay knowledge about aggression. Most respondents do not understand the basic concepts: "aggression", "aggressiveness", "aggressive behavior". Students associate aggressiveness with the emotional state or manifestation of negative emotions which does not correspond to the scientific definition of aggressiveness as a psychological feature of a person whose manifestation 
depends on certain factors and can take a socially acceptable or destructive form: harm to others, resentment, damage. In order to assist adolescents with aggressive behavior, the teacher must have relevant knowledge and possess a number of special skills.

In general, the results of the ascertaining experiment allow us to draw the following conclusions: the system of training students for work with adolescents needs to be changed focusing students on interaction with adolescents; it is necessary to improve the theoretical and practical levels of professional training, taking into account modern achievements of pedagogical and psychological science studying "difficult" childhood, teenage aggressiveness.

The results of the ascertaining experiment made it possible to organize the experimental work on the development of professional readiness for working with children exhibiting aggressive behavior.

The main forms, methods and techniques that contribute to the implementation of pedagogical conditions and ensure development of readiness to work with aggressive teenagers are students of the integrated training course "Organization of work with aggressive children and adolescents"; organization of practical classes with students aimed at developing relevant skills; solution of psychological and pedagogical problems; participation in research activities.

We suggested that the implementation of a set of pedagogical conditions will ensure development of readiness for working with aggressive teenagers. The hypothesis was verified during the pedagogical experiment. A modern teacher having mastered knowledge of developmental features of adolescents with aggressive behavior, available technologies for diagnostic and correctional work can help them. The integrated training course "Organization of work with aggressive children and adolescents" was introduced into the curriculum. It represents a theoretical and practical block of the program for developing professional readiness to work with aggressive adolescents.

The main objective of the training course is to form integrated knowledge about diagnostic, educational and correctional work with adolescents exhibiting aggressive behavior. Based on the goal, a number of important tasks were solved:

- formation of a system of professional knowledge about interaction with adolescents exhibiting aggressive behavior based on the principles of humane pedagogy;

- mastering skills for conducting the diagnostic work with adolescents aimed at the timely identification of behavior deviations; formation of the ability to provide pedagogical assistance to children;

- mastering technologies for conducting the corrective work with adolescents exhibiting aggressive behavior.

The curriculum includes 5 topics:

1. Modern scientific views on teaching children exhibiting deviant behavior. The problem of aggressiveness of children and adolescents and its solution in psychological and pedagogical sciences.

2. Aggressive behavior of modern children and adolescents (causes, specifics of manifestation, types).
3. Diagnostics of aggressiveness of children and adolescents (methods for studying aggressiveness of adolescents and children).

4. Psychological and pedagogical prevention of aggressive behavior of children and adolescents: general principles, conditions, rules and technologies.

5. Psychological and pedagogical correction of aggressive behavior: general principles, conditions, rules and technologies.

Topics 1 and 2 reveal the essence of modern scientific theories of aggressive behavior, the specifics of manifestation of aggressive behavior, conditions and characteristics of aggressive behavior. Topics 3-5 focus on the specifics of activities of teachers aimed at diagnosing, preventing and correcting aggressiveness of children and adolescents. The curriculum provides for 58 hours, of which: lectures - 30 hours, seminars and workshops - 28 hours. The training course contributes to the formation of methodological and theoretical knowledge which is part of the cognitive component of professional readiness to work with aggressive teenagers. For each section of the curriculum, theoretical and practical classes were planned.

Theoretical classes included the study of issues provided for by each topic, compulsory and additional literature. An analysis of literature on pedagogical correction of deviant behavior of adolescents showed that there are no textbooks that integrate modern achievements of science. In studying scientific views on deviant behavior, much attention was paid to the main types and forms of behavior of children, social causes of deviant behavior and aggressiveness. Many students themselves lacked a normal psychological climate in their families.

The seminars and workshops were aimed at consolidating theoretical knowledge, developing an ability to put it into practice. A variety of teaching methods were used. As didactic tools for the formation of cognitive, motivational-personal and practical components of professional readiness for corrective work with aggressive teenagers, we used psychological and pedagogical tasks, imitation, role-playing games and exercises.

There were two control sections of knowledge which made it possible to establish the dynamics of formation of the cognitive component. A high level was observed in $28 \%$ of students, medium - in $57 \%$ and low - in $15 \%$. The work allowed us to change the traditional negative attitude towards adolescents with behavioral abnormalities and aroused interest in studying methods for correcting teenage aggressiveness. $31 \%$ of students realized the importance of the correctional work with aggressive teenagers (high level), the average level of formation of the motivational-personal component was observed in $55 \%$ of students and the low level was observed in $14 \%$.

The technology unit of development of the professional readiness presented by the model helped students master the technology of pedagogical diagnostics and correction of aggressive behavior, form initial experience of elimination of aggressive behavior [9].

As part of the formative experiment, 2nd, 3rd and 4th year students of the Faculty of Pedagogy and Psychology of Nizhnevartovsk State University conducted research 
during their internships. They studied the school system for correcting aggressive behavior, analyzed this work in terms of its effectiveness, complexity, scientific validity; diagnosed aggressiveness of adolescents and their parents in order to identify the nature of family education, the communication style of children and parents, the dominant type of family education; delivered lectures for parents on relevant psychological, pedagogical and medical-social problems of adolescents; participated in seminars for school teachers on relevant psychological, pedagogical and medical-social problems of adolescents; participated in psychological and pedagogical trainings for parents based on a system of special pedagogical situations that reproduce various aspects of conflict situations in the communication of children and parents. Taking a direct part in diagnosing adolescents with manifestations of aggression, students mastered the method of its implementation and faced with results that made them want to deepen their knowledge about deviant behavior of children and manifestations of aggression. Students consolidated knowledge about various means: arts (artworks, music, drawing), games and technical means (video films, audio recordings of musical works). The latter two means raised the greatest interest in adolescents due to their age characteristics.

One of the conditions for the development of professional readiness for working with aggressive teenagers was participation of students in research activities. The interest and desire for a deeper study of the problem is reflected in the topics of abstracts, term papers and dissertations: "Ways to overcome physical aggression in adolescents"; "The role of game in correcting aggressive behavior of adolescents"; "Effective forms and methods of work of a social educator with adolescents exhibiting aggressive behavior"; "Effective forms and methods of work of a school psychologist with adolescents exhibiting aggressive behavior"; Involvement of aggressive adolescents in the system of socially recognized and socially approved activities as a condition for correction of the aggressive behavior; "The influence of the media on deviations in the behavior of adolescents"; "The effect of punishment on behavior deviations in adolescent children"; "Ways of forming an emotional well-being for teenage children"; " Implementation of the spiritual experience of the Khanty and Mansi peoples in educational work to prevent and eliminate aggressive behavior of adolescents." These works were highly appreciated at the scientific and practical conferences of the university. In the first two topics, origins of teenage aggression, age-related forms of its manifestation were studied, possibilities of using corrective means were investigated. Students selected various games, exercises combined with copyright findings. One of the forms of implementing practical and motivational-personal components of readiness was participation of students in the scientific community.

To determine the dynamics of growth of indicators of readiness levels, we conducted a repeated survey of students, control of knowledge, analysis of theses, term papers, and creative works.

Assessing the level of formation of the practical component of student's readiness to work with aggressive teenagers, we observed positive changes associated with independence and awareness of using organizational, diagnostic and correctional skills in practical activities. A high level of formation of the practical component was observed in 42 students (39\%), medium - in 55 students $(52 \%)$, and low - in 10 students $(9 \%)$. Comparative results are presented in Table 1.

Table 1. Comparative results of the pedagogical experiment

\begin{tabular}{|l|c|c|c|c|}
\hline \multirow{2}{*}{$\begin{array}{l}\text { Level of readiness } \\
\text { for work with }\end{array}$} & $\begin{array}{l}\text { The ascertaining } \\
\text { lage of the }\end{array}$ experiment & \multicolumn{2}{|l|}{$\begin{array}{l}\text { The control stage } \\
\text { of the experiment }\end{array}$} \\
\cline { 2 - 5 } aggressive teenagers & people & $\%$ rel. & people & $\%$ rel. \\
\hline High & 17 & $16 \%$ & 42 & $39 \%$ \\
\hline Medium & 39 & $36 \%$ & 55 & $52 \%$ \\
\hline Low & 51 & $48 \%$ & 10 & $9 \%$ \\
\hline Total & 107 & $100 \%$ & 107 & $100 \%$ \\
\hline
\end{tabular}

Comparison of results of the development dynamics for professional readiness allows us to state that there were quantitative changes in the professional readiness to work with aggressive teenagers: the number of students who had a low level of readiness decreased by $41 \%$; the number of students with a high level increased by $16 \%$; the number of students with an average level of readiness increased by $25 \%$.

The main research tasks were solved and experimental data confirming the validity of the hypothesis were obtained. The results allow for the conclusion that students are ready to work with aggressive adolescents. Development of students' readiness for interaction with adolescents characterized by aggressive behavior should involve a relationship between the disciplines of psychological, pedagogical and special cycles during which students can obtain integrated knowledge about the nature of aggressiveness in adolescents. The practiceoriented nature of professional training helps students master the technology of pedagogical diagnosis and correction of aggressive behavior of adolescents. The use of active forms and teaching methods, as well as research activities helps increase the effectiveness of formation of students' professional readiness to work with aggressive teenagers.

\section{References}

1. K.M. Duray-Novakova Formation of professional readiness of students to teaching, 386, (1983)

2. L. Ibragimova, I. Skobeleva. Conditions for creation and development of media culture in students of secondary professional education. Media education. 1. 89-94, (2018).

3. R. S Nagovitsyn., I. A Golubeva. The formation of communicative competencies of future teachers in the student scientific society Integration of Education, 23, 1, 66-84. (2019)

4. V.N. Irkkhin Stimulating the orientation of future teachers with pedagogically neglected adolescents, 16 (1991) 
5. V.V. Trifonov Training future teachers to work with difficult students, 231, (1991)

6. A. Bandura, R. Walters. Teenage Aggression: A Study of the Impact of Parenting and Family Relations, 508. (April, Press, 1999)

7. O.I. Istrofilova Pedagogical conditions for formation of professional readiness of future teachers to work with adolescents exhibiting aggressive behavior:
Thesis, Moscow State Open Pedagogical University n.a. M.A. Sholokhov, 2 6, 2006

8. T.Ya. Yakovets Pedagogical conditions for the formation of readiness of university students for selfeducation 23 ( Kurgan, 1999)

9. O.I. Istrofilova Organization of work to correct aggressive behavior of adolescents in school. European Social Science Journal. 5-2 (44). 153-158. (2014) 